\title{
Transdifferentiation of type II alveolar epithelial cells induces reactivation of dormant tumor cells by enhancing TGF-B1/SNAI2 signaling
}

\author{
YUANHONG ZHOU*, QIANG LIU*, XIANGCHEN DAI, YULING YAN, YING YANG, \\ HUA LI, XIAOLINGZHOU, WENQI GAO, XIAOLAN LI and ZUYANG XI
}

The First College of Clinical Medical Science, China Three Gorges University/Yichang Central People's Hospital, Yichang, Hubei 443000, P.R. China

Received July 28, 2017; Accepted January 5, 2018

DOI: 10.3892/or.2018.6247

\begin{abstract}
Dormant tumor cells (DTCs) serve a crucial role in the pathogenesis of metastasis and may compromise the efficacy of existing therapeutic modalities aimed at fully eradicating cancer. However, the mechanisms underlying the dormant-to-proliferating switch of DTCs remain largely unknown. The lung is one of the most common sites of metastatic recurrence. The transdifferentiation of alveolar epithelial cells II (AEC IIs) to AEC Is is a hallmark of alveolar epithelial stimulation. However, the role of AEC II transdifferentiation during the reactivation of DTCs has not been fully elucidated. In the present study, we found that tumor cells promoted the transdifferentiation of AEC IIs. Furthermore, the supernatant of the transdifferentiation of AEC IIs to AEC Is (Super-TDA) promoted the dormant-to-proliferating switch of DTCs via the autocrine effect of TGF- $\beta 1$ on the 3D BME culture system in vitro. Monensin and LY2109761 blocked the dormant-to-proliferating switch of DTCs induced by Super-TDA. Although lipopolysaccharide did not directly stimulate the reactivation of DTCs, it promoted DTC reactivation by increasing the secretion of TGF- $\beta 1$ in the Super-TDA. We further demonstrated that the upregulation of SNAI2 expression was required for Super-TDA facilitating the DTC
\end{abstract}

Correspondence to: Professor Zuyang Xi, The First College of Clinical Medical Science, China Three Gorges University/Yichang Central People's Hospital, Yichang, Hubei 443000, P.R. China E-mail: zyh205720@sohu.com

*Contributed equally

Abbreviations: Super-TDA, supernatant of the transdifferentiation of AEC IIs to AEC Is; Super-CTA, supernatant of co-cultured tumor cells and AEC IIs by Transwell assay; AEC IIs, type II alveolar epithelial cells; 3D, three dimensional; DTC, dormant tumor cell; BME, Cultrex growth factor-reduced basement membrane extract; LPS, lipopolysaccharide

Key words: DTCs, transdifferentiation, AEC, dormant-toproliferating switch, SNAI2 dormant-to-proliferating switch. Taken together, our findings demonstrated that tumor cells may promote AEC II transdifferentiation. Furthermore, the transdifferentiation of AEC IIs may, in turn, induce the reactivation of 3D-established DTCs by promoting TGF- $\beta 1 / \mathrm{SNAI} 2$ signaling. Targeting this process may provide novel therapeutic strategies for the inhibition of the dormant-to-proliferating switch.

\section{Introduction}

Dormant solitary cells, or minimal dormant metastases, have been recognized as the main cause of cancer recurrence (1-3). Tumor dormancy is maintained in a microenvironment unfavorable for tumor cell proliferation $(3,4)$. When the microenvironmental conditions shift to support tumor expansion, dormant tumors may resume active growth and progression (3). Therefore, understanding the mechanisms that regulate dormancy or the switch to a proliferative state is crucial for identifying novel targets and designing interventions to prevent disease recurrence.

Compared with highly invasive human cancer cells, non-invasive human cancer cells constitutively have a less prominent malignant phenotype, as the metastatic tumor cells derived from non-invasive tumor cells may be more sensitive to the restriction of a new microenvironment (5). Our previous study demonstrated that tumor cells seeded at metastatic sites have a lower proliferative potential, and remain quiescent over long periods of time (5). Following a period of dormancy in metastatic sites, dormant tumor cells (DTCs) may ultimately transit through an angiogenic switch and become clinically apparent metastases $(5,7)$, whose size or proliferative potential is limited by the lack of a favorable tumor environment (6), suggesting that the microenvironment serves a key role in tumor cell dormancy. However, whether tumor dormancy is a consequence of deficiency or alteration of the proper growth signals in the secondary target site require further investigation.

Metastasis and recurrence is the leading cause of death in patients suffering from cancer, and the lung is the one of most common sites of metastasis (8). Alveolar epithelial cells II (AEC IIs) serve a multifunctional role in the lung, including 
secretory, synthetic and remodeling reservoirs for the lung epithelium to host defense (9). The AEC IIs are the progenitors of AEC Is, and the progenitor function of AEC IIs may be activated when the lung epithelium encounters a variety of stimulators, including acute lung injury or bacteria, among others, to defend the alveolus from injury $(10,11)$. However, whether the tumor cells metastasizing to the lung are able to stimulate AEC II transdifferentiation has not been examined in detail.

Transforming growth factor $\beta 1$ (TGF- $\beta 1$ ) is a key factor in the alteration of the tumor environment (14), that is secreted during the transdifferentiation of AEC IIs to AEC Is $(12,13)$; these alterations in the tumor environment may be implicated in the regulation of a variety of biological responses, including cell proliferation and differentiation (14). The lung is one of most common sites of metastasis (8). However, the role of AEC II transdifferentiation during reactivation of DTCs has not been fully elucidated.

In the present study, we investigated whether tumor cells can stimulate the transdifferentiation of AEC IIs into AEC Is, as well as whether AEC II transdifferentiation can induce reactivation of DTCs in the lung. Our results revealed that tumor cells may promote the transdifferentiation of AEC IIs into AEC Is. Furthermore, the transdifferentiation of AEC IIs may, in turn, promote the switch of metastasized DTCs to reactivate growth via TGF- $\beta 1$, secreted by AEC II transdifferentiation, leading to increase the SNAI2 expression in DTCs. Targeting this process may provide novel therapeutic strategies for the inhibition of the dormant-to-proliferating metastatic switch.

\section{Materials and methods}

Ethics statement. All animal experiments were conducted according to the relevant national and international guidelines, and were approved by the Ethics Committee on Animal Experiments of the China Three Gorges University and monitored by the Department of Experimental Animals of the Yichang Central People's Hospital.

Cells and reagents. The human breast cancer cell lines MCF-7 and T-47D were purchased from the Type Culture Collection of the Chinese Academy of Sciences (Shanghai, China), and maintained in Dulbecco's modified Eagle's medium (DMEM) with high glucose, $10 \%$ fetal bovine serum (FBS) and antibiotics (Life Technologies, Carlsbad, CA, USA), according to the guidelines. Lipopolysaccharide (LPS) was purchased from Sigma-Aldrich (St. Louis, MO, USA). Growth factor-reduced 3D Cultrex basement membrane extract (BME) was purchased from Trevigen Inc. (Gaithersburg, MD, USA). Monensin was purchased from Selleckchem.cn. Antibodies against surfactant protein $\mathrm{C}$ (SP-C) and aquaporin 5 (AQP5), and all secondary antibodies, were purchased from Santa Cruz Biotechnology (Santa Cruz, CA, USA) and Cell Signaling Technology Inc. (Beverly, MA, USA). The TGF- $\beta$ receptor kinase inhibitor LY2109761 was purchased from Selleckchem.cn.

Gene expression assessment by reverse transcription-quantitative polymerase chain reaction $(R T-q P C R)$. Total RNA was extracted from cells with TRIzol ${ }^{\circledR}$ reagent (Invitrogen, Carlsbad, CA, USA). Total RNA (100 ng) was used for reverse transcription using SuperScript II RNase H reverse transcriptase (Invitrogen) in a volume of $25 \mu \mathrm{l}$. Then, $1 \mu \mathrm{l}$ of cDNA was amplified with SYBR-Green Universal PCR Master Mix (Bio-Rad, Richmond, CA, USA) in duplicate. Quantification of the expression of genes was performed using the comparative $\mathrm{C}_{\mathrm{T}}$ method (Sequence Detector User Bulletin 2; Applied Biosystems, Carlsbad, CA, USA). The expression level of each mRNA was normalized to GAPDH mRNA and expressed as n-fold difference relative to the control (calibrator). The PCR was conducted using the following parameters: $95^{\circ} \mathrm{C}$ for $5 \mathrm{~min}$, and 40 cycles at $95^{\circ} \mathrm{C}$ for $10 \mathrm{sec}, 60^{\circ}$ for $20 \mathrm{sec}$ and $72^{\circ} \mathrm{C}$ for $15 \mathrm{sec}$. The relative quantity of mRNA was determined by RT-qPCR, as previously described (15). The mRNA of GAPDH was used as the internal control. The primer sequences were as follows: SNAI2 sense, 5'-AGG AATCTGGCTGCTGTG-3' and antisense, 5'-GGAGAAAAT GCCTTTGGAC-3'; SP-C sense, 5'-GTCGTCGTGGTGATT GTAGGG-3' and antisense, 5'-GAAGGTAGCGATGGTGT CTG-3'; AQP5 sense, 5'-CGTCAATGCGCTGAACAACAA-3' and antisense, 5'-ACAGACAAGCCAATGGATAAGG-3'; GAPDH sense, 5'-TCATTGACCTCAACTACATGGTTT-3' and antisense, 5'-GAAGATGGTGATGGGATTTC-3'.

Western blot assay. Western blotting was performed as previously described (5). Cell lysates (30 $\mu \mathrm{g}$ of total protein) and prestained molecular weight markers were separated by sodium dodecyl sulfate-polyacrylamide gel electrophoresis (SDS-PAGE) followed by the transfer onto nitrocellulose membranes. The membranes were blocked in Tris-buffered saline with $0.5 \%$ Triton X-100 (TBST) containing 5\% non-fat milk and probed with antibodies (SPC, sc-13979, 1:1,000; AQP5, sc-514022, 1:1,000; SNAI2, \#9585, 1:1,000; $\beta$-actin, sc-58673, 1:5,000) at $4^{\circ} \mathrm{C}$ with gentle shaking, overnight. After incubation with the secondary antibody (sc-2357, 1:10,000; sc-516102, 1:10,000) conjugated with horseradish peroxidise at room temperature with gentle shaking for $2 \mathrm{~h}$, membranes were extensively washed, and the immunoreactivity was visualized by enhanced chemiluminescence according to the manufacturer's protocol (ECL kit; Santa Cruz Biotechnology). Antibodies were purchased from Santa Cruz Biotechnology and Cell Signaling Technology. The protein expression data were analyzed using Image J software (NIH; National Institutes of Health, Bethesda, MD, USA).

Isolation and culture of AEC IIs. AEC IIs were isolated from pathogen-free Kunming (KM) mice (20-25 g) by an improved method (16). The animal experimental protocols were approved by the Ethics Committee of China Three Gorges University. Mice were acclimated to the laboratory conditions, with free access to food and water in a facility with controlled temperature $\left(22^{\circ} \mathrm{C}\right)$ on a $12 / 12$-h light/dark cycle, for 1 week prior to the experiments. In brief, adult mouse lungs were lavaged with PBS, and then digested with elastase $(1 \mathrm{mg} / \mathrm{ml})$. The cell mixture was filtered through a $200-$ and a $100-\mu \mathrm{m}$ nylon mesh (twice through each), plated on mouse IgG-coated plates and incubated at $37^{\circ} \mathrm{C}$ for 3-4 h to remove macrophages and other pulmonary cells containing an Fc-fragment. The purity of AEC IIs was $>96 \pm 1.8 \%$, as determined by modified Papanicolaou staining, and the viability was $>98 \pm 2.1 \%$. The detailed protocols were applied as previously described (16). 
Transdifferentiation of AEC IIs. AEC IIs were seeded onto 6 -well plastic culture dishes at a density of $1.5 \times 10^{6}$ cells/dish, and cultured in media with $10 \%$ FBS with or without breast tumor cells $\left(5 \times 10^{3}\right.$ cells/dish), with the cells contacting each other (co-cultured contact) or divided with a Transwell assay (co-cultured no contact). The media were changed after the first $24 \mathrm{~h}$, and on alternate days thereafter. The level of TGF- $\beta 1$ and other cell factors secreted into the media were determined using ELISA kits from R\&D Systems (Minneapolis, MN, USA). AEC Is are the components of the alveolar epithelium most susceptible to injury. Following lung injury, AEC Is are destroyed and AEC IIs proliferate and differentiate into AEC Is to repair the epithelium (17). Therefore, this in vitro transdifferentiation model may be closely associated with the process occurring in vivo during lung injury and repair. It should also be noted that isolating AEC IIs from the lung and culturing them may mimic lung disturbance and repair.

Preparation of super-TDA. The transdifferentiation of AEC IIs to AEC I-like cells was evident from day 3 onwards and complete by day 5 , as shown by the present study, as well as previous data $(16,29)$. AEC IIs were seeded onto 6-well plastic culture plates at a density of $1.5 \times 10^{6}$ cells/dish and cultured in media with $10 \%$ FBS for 4 days, after which time the medium was replaced with fresh medium $(1 \mathrm{ml})$ without FBS and the cells were incubated for a further $24 \mathrm{~h}$, followed by harvesting of the supernatants from the cultures. The supernatants were then passed through a $0.22-\mu \mathrm{m}$ filter and stored at $-80^{\circ} \mathrm{C}$ until further use.

An in vitro $3 D$ system inducing tumor dormancy. The cell culture system was pre-coated with an adequate amount of Cultrex growth factor-reduced BME (protein concentration, $14-15 \mathrm{mg} / \mathrm{ml}$; thickness, $1-2 \mathrm{~mm}$ ) in a humidified incubator with $5 \% \mathrm{CO}_{2}$ at $37^{\circ} \mathrm{C}$ for $30 \mathrm{~min}$; the culture plates were then rinsed 3 times with $10 \mathrm{ml}$ PBS (pH 7.4) for further use. The tumor cells were suspended in a mixture of $2 \%$ FBS and $2 \%$ Cultre ${ }^{\circledR}$ DMEM mixture (CFD). The cells were cultured on the pre-coated slides and re-fed every 2 days (29), and the proliferative ability of the cells was then determined via an MTT assay and flow cytometry.

MTT proliferation assay. Plates with 96-well were coated with 50-100 $\mu$ l BME. Non-invasive MCF-7 or T-47D cells $\left(1.5 \times 10^{3}\right.$ to $2.0 \times 10^{3} /$ well) were re-suspended in $200 \mu \mathrm{l}$ CFD and grown on the BME pre-coated plates. The Cell Titer 96 Aqueous One Solution cell proliferation assay kit (Promega Corporation, Madison, WI, USA) was used to measure cell proliferation according to the manufacturer's instructions.

Cell cycle analysis by flow cytometry. The tumor cells $\left(2.5 \times 10^{5} /\right.$ well $)$ were cultured under $3 \mathrm{D}$ conditions in the presence of Super-TDA (50\%) for 5 days; the tumor cells were then analyzed using propidium iodide (PI; Molecular Probes, Invitrogen) to distinguish non-viable cells. DNA synthesis or the total DNA content was measured by flow cytometry using a FACSCalibur flow cytometer (BD Accuri ${ }^{\mathrm{TM}}$ C6).

Cell transfection. For the downregulation of SNAI2, MCF-7 cells were transduced with SNAI2 shRNA lentiviral particles, or control shRNA lentiviral particles (Santa Cruz Biotechnology) according to the manufacturer's protocol. Following selection with puromycin, the cells were used for further experiments.

Statistical analysis. Data were pooled from 3 independent experiments with a total of 6 samples in each group. Results are expressed as the mean $\pm \mathrm{SD}$ and interpreted by one-way ANOVA. Differences were considered to be statistically significant when $\mathrm{P}<0.05$.

\section{Results}

Tumor cells promote transdifferentiation of AEC IIs into $A E C$ Is in vitro. The isolated AEC IIs were seeded on plastic dishes and cultured for several days. The transdifferentiation of AEC IIs to AECI-like cells was evident from day 3 onwards and was completed by day 5 , as previously shown (16). When the isolated AEC IIs $\left(1.5 \times 10^{6} /\right.$ well $)$ were co-cultured in the presence of tumor cells (MCF-7 or T-47D, $5 \times 10^{3} /$ well), morphometric observation of isolated AEC IIs discerned characteristics almost typical of AEC Is at 3 days. To further characterize the cell phenotype, qPCR and western blotting with specific labels generated to the AEC IIs phenotypic marker SP-C (18) and to the AEC Is phenotypic marker AQP5 (18) were used. Our results revealed that the AEC IIs exhibited a significant decrease in SP-C mRNA expression (Fig. 1A) and a significant increase in AQP5 mRNA expression (Fig. 1B) when co-cultured with tumor cells (MCF-7 and T-47D) for 3 day. Furthermore, western blot analysis revealed that SP-C protein expression decreased and AQP5 protein expression increased in AEC IIs over time (Fig. 1B). In addition, the changes during AEC II transdifferentiation did not differ between the co-culture contact and co-culture no contact group. The transition images of AEC IIs to AEC Is is shown in Fig. 1C. Therefore, our results demonstrated that the tumor cells promoted AEC II transdifferentiation into AEC Is in vitro.

Establishment of solitary DTC model in vitro. Cell dormancy may be defined as a non-proliferative state or an arrested stage in the cell cycle that results in a prolonged G0 phase. Due to their small size and non-invasive nature, these DTCs remain asymptomatic and, in most cases, undetected. Unfortunately, these DTCs are resistant to conventional therapies targeting actively dividing cells, which likely accounts for disease recurrence following apparent successful treatment of the primary tumors $(19,20)$. However, the reasons for the activation of DTCs are not clear, mainly due to lack of a DTC model.

The 3D BME culture system was used as a model for DTCs, as described above. MCF-7 cells exhibit dormant behavior when cultured in the 3D BME system, consistent with their behavior at distant sites in vivo $(6,21)$. In the present study, we successfully established a solitary DTC model for MCF-7 and T-47D cells. We found that both MCF-7 and T-47D cells did not proliferate, and remained quiescent throughout the entire experimental 18-day culture period when cultured in 3D BME $(\mathrm{P} \leq 0.01)$. In addition, both breast cancer cell lines proliferated readily when cultured on a 2D plastic substrate (Fig. 2A).

We further investigated the cell cycle of dormant MCF-7 and T-47D cells using flow cytometry. Our results revealed 
A
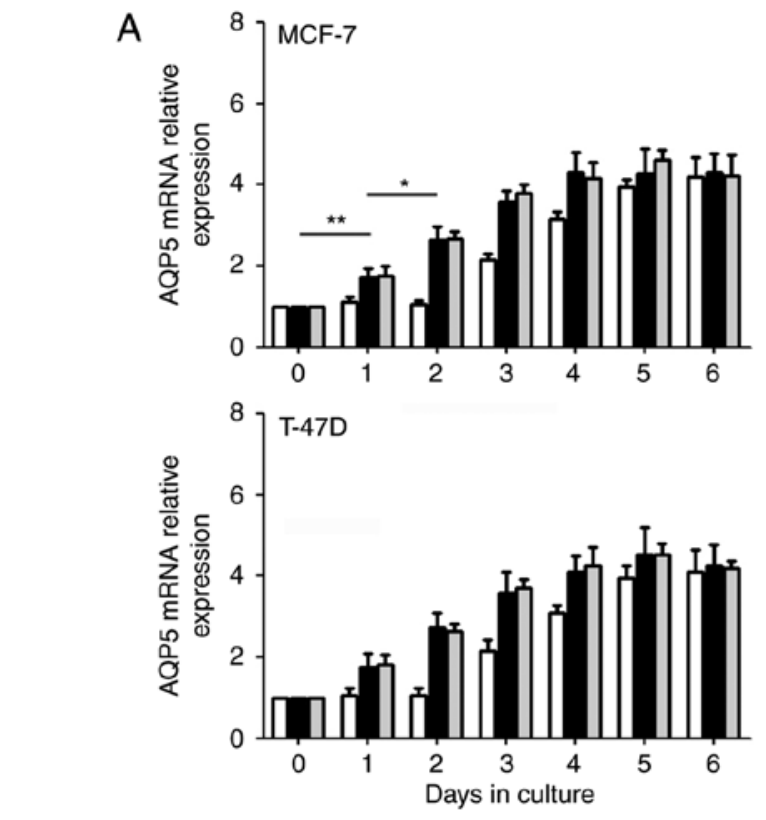

B

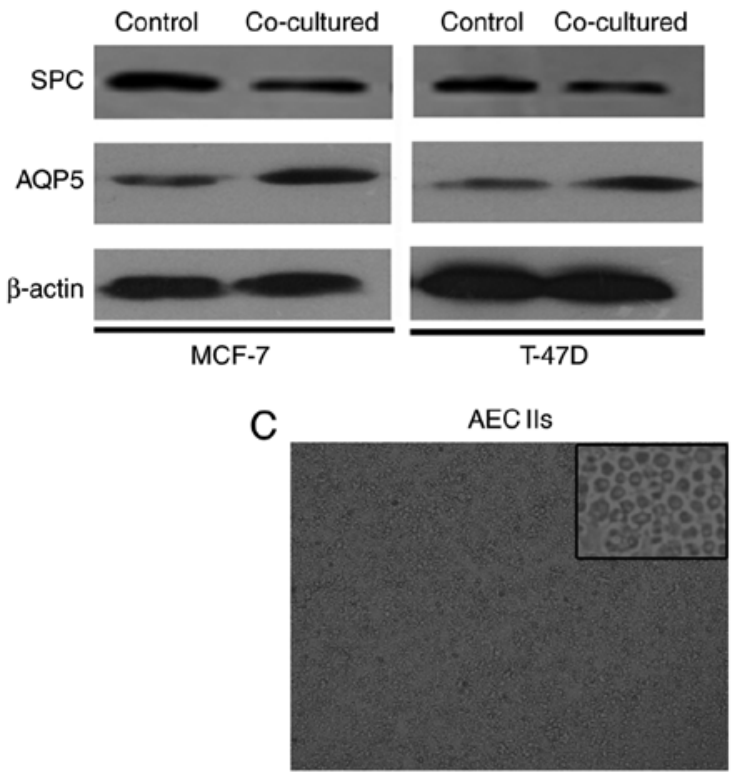

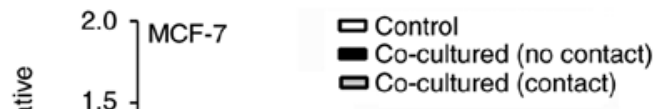

Do-cultured (contact)
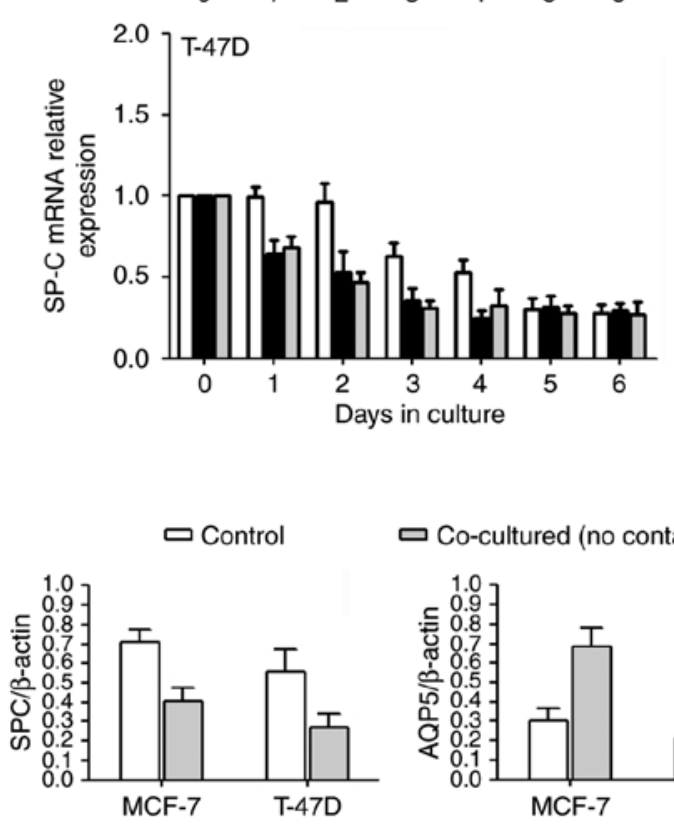

$\square$ Co-cultured (no contact)

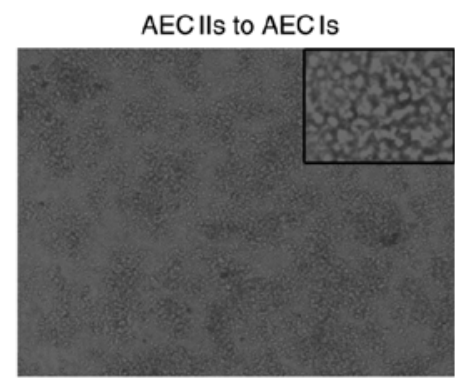

Figure 1. Tumor cells promote the transdifferentiation of AEC IIs to AEC Is. (A and B) Tumor cells (MCF-7 and T-47D) enhanced the decrease in SP-C expression, and the increase in AQP5 expression in AEC IIs. ${ }^{*} \mathrm{P}<0.05,{ }^{* *} \mathrm{P}<0.01$. (C) Representative images of AEC II and transdifferentiation of AEC IIs to AEC Is. SP-C, surfactant protein C; AQP5, aquaporin 5; AEC IIs, type II alveolar epithelial cells; AEC Is, type I alveolar epithelial cells.

that a high percentage of MCF-7 and T-47D cells remained in the $\mathrm{G} 0 / \mathrm{G} 1$ phase $(\mathrm{P} \leq 0.01)$, with a smaller $\mathrm{S}$ and $\mathrm{G} 2 / \mathrm{M}$ cell population $(\mathrm{P} \leq 0.01)$, when cultured under $3 \mathrm{D}$ conditions for 5 days, suggesting that the cells remained at an arrested stage of a prolonged G0 phase (Fig. 2B).

In brief, MCF-7 and T-47D cells were quiescent when cultured in the 3D BME system (3D).

The supernatant of transdifferentiation of AEC IIs to AEC Is (Super-TDA) may promote DTC growth by altering the cell cycle. To determine whether Super-TDA induces a dormant-to-proliferating switch of the DTCs, DTCs were cultured with the Super-TDA (50\%), and we observed that the DTCs were reactivated and proliferated stably (Fig. 3A). We further investigated whether the Super-TDA affected the cell cycle of dormant MCF-7 and T-47D cells using flow cytometry. Our results revealed that a high percentage of MCF-7 and T-47D cells remained in G0/G1 when cultured under 3D conditions for 5 days (Fig. 3B). However, the established-DTCs exhibited a significantly higher $\mathrm{S}$ and $\mathrm{G} 2 / \mathrm{M}$ cell population when co-cultured with Super-TDA $(50 \%)$ for 5 days, compared with parental cells $(\mathrm{P} \leq 0.01)$. In conclusion, Super-TDA promoted the dormant-to-proliferating switch by altering the cell cycle.

TGF- $\beta 1$ secreted during the transdifferentiation of AEC IIs is one of the major stimulants involved in inducing DTC reproliferation. TGF- $\beta 1$ is a key factor altering the tumor environment, and is implicated in the regulation of a variety of biological responses (14), including proliferation and differentiation of tumor cells. To further identify factors involved in inducing the growth of DTCs in Super-TDA, TGF- $\beta 1$ 


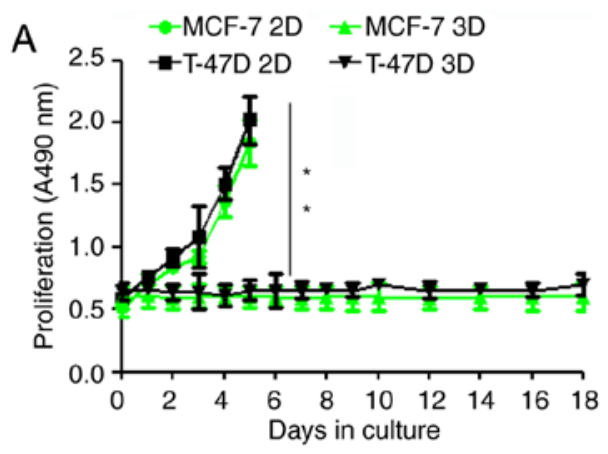

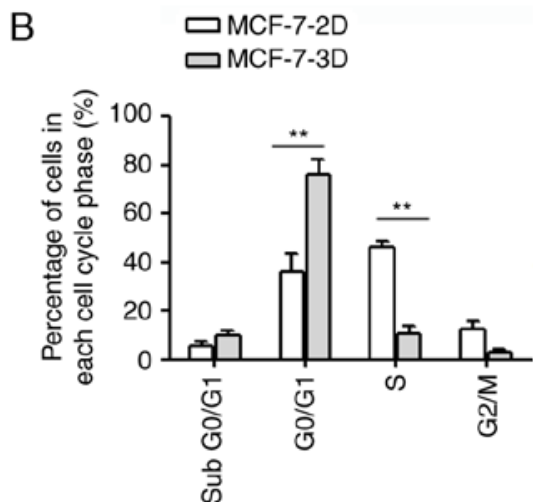

Cell cycle phase

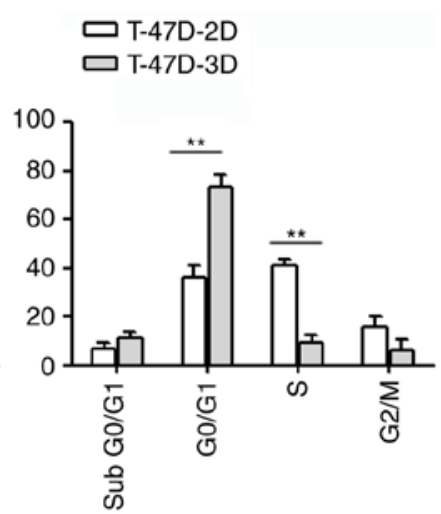

Cell cycle phase

Figure 2. Establishment of the DTC model in vitro. (A) The proliferative potential of MCF-7 and T-47D cells was examined by MTT assay in 2 and 3D culture systems (MCF-7 2D, T-47D 2D, MCF-7 3D and T-47D 3D). ${ }^{* *} \mathrm{P} \leq 0.01$. (B) Flow cytometry cell cycle analysis in MCF-7 and T-47D cells treated under 2D and 3D culture conditions using PI as a non-viable DNA dye. ${ }^{* *} \mathrm{P} \leq 0.01$. DTC, dormant tumor cell; PI, propidium iodide.

A

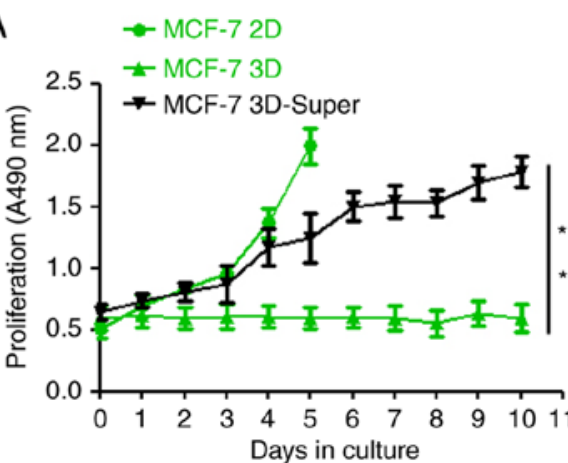

$\mathrm{B}$

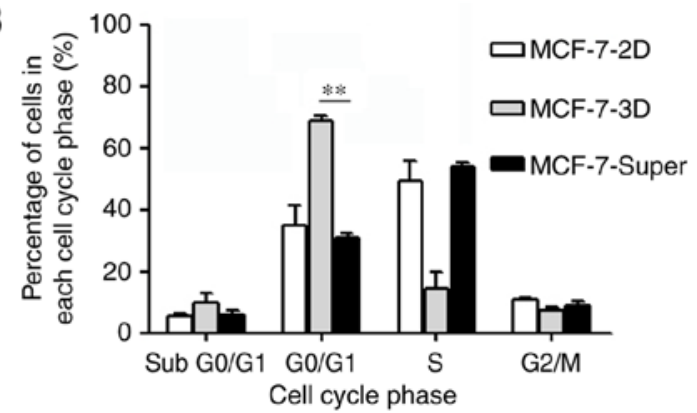

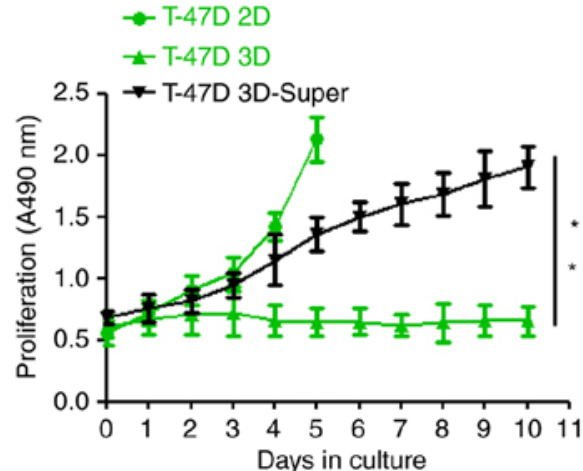

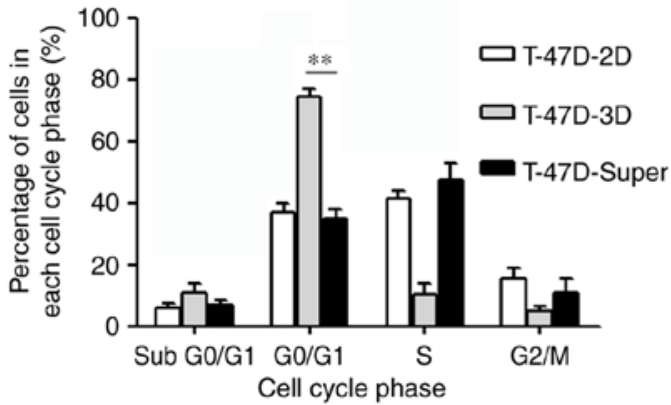

C

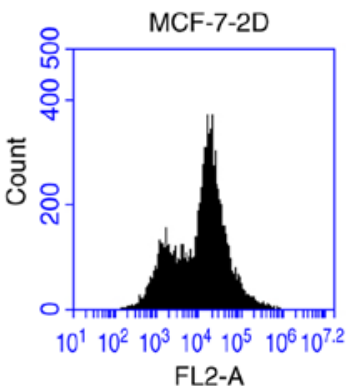

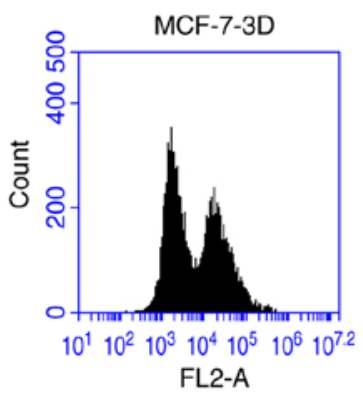

FL2-A

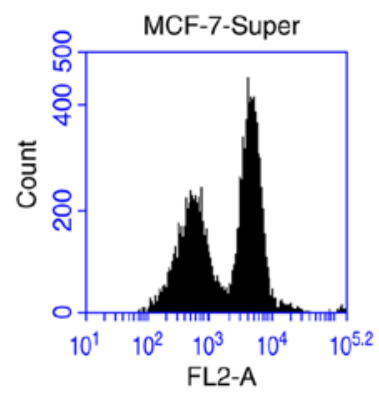

Figure 3. Super-TDA promotes the dormant-to-proliferating switch of DTCs. (A) The proliferative potential of breast cancer cells was assessed using the MTT assay in 2D and 3D culture systems with ordinary medium or Super-TDA (50\%) (MCF-7 3D-Super, T-47D 3D-Super). (B) Flow cytometry cell cycle analysis in MCF-7 and T-47D cells treated with Super-TDA (50\%) under 2D and 3D culture conditions using PI as a non-viable DNA dye. ** P $\leq 0.01$. (C) Representative images of cell phase. DTCs, dormant tumor cells; Super-TDA, supernatant of the transdifferentiation of AEC IIs to AEC Is; PI, propidium iodide.

expression was evaluated by sandwich ELISA (Fig. 4). Our data revealed that the level of secreted-TGF- $\beta 1$ was markedly increased with the progression of the transdifferentiation of
AEC IIs to AEC Is, particularly on day 5 , and the amount of TGF- $\beta 1$ were nearly 12 times that in the control group $(\mathrm{P} \leq 0.01)$ (Fig. 4A). Furthermore, we observed that the amount 

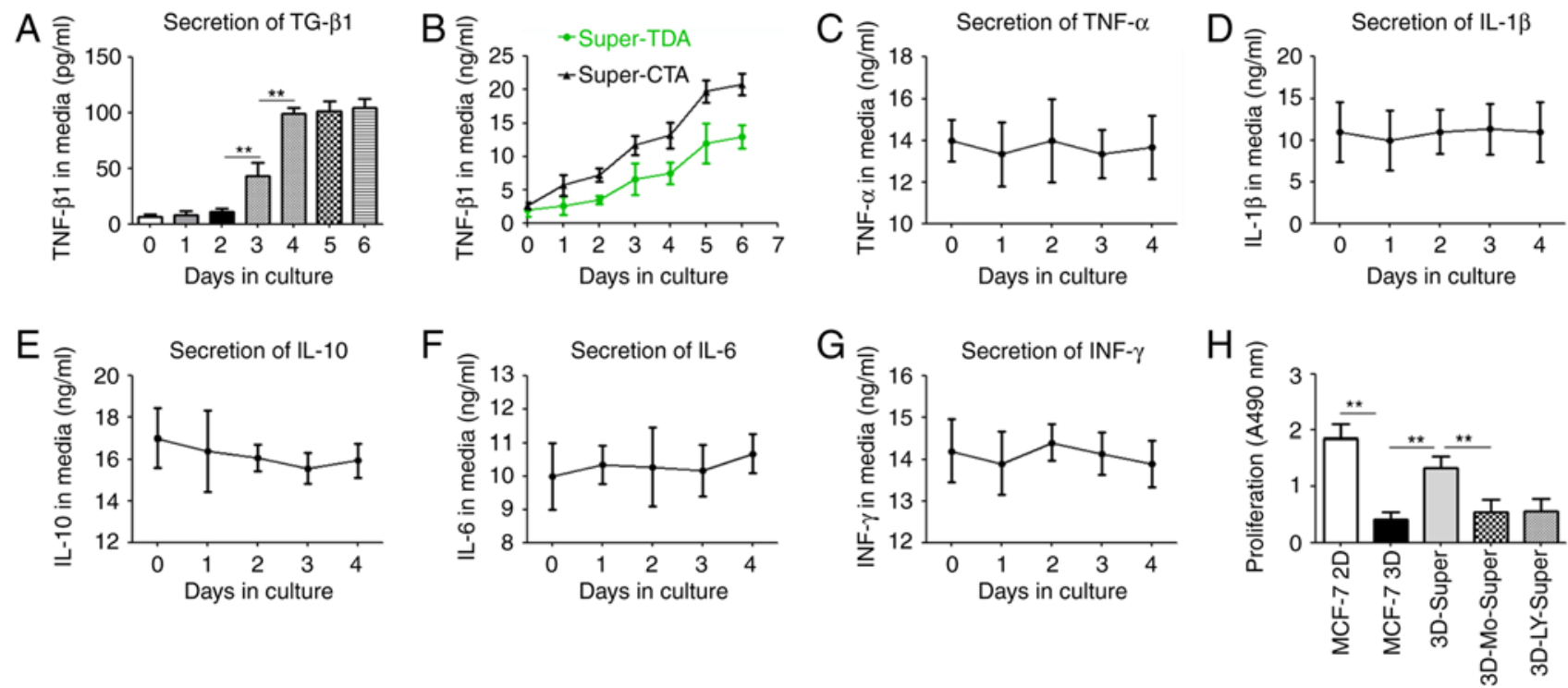

Figure 4. The autocrine effect of TGF- $\beta 1$ is a major stimulator inducing DTC growth. (A and C-G) The amount of TGF- $\beta 1$ and other cell factors in Super-TDA $(50 \%)$ were demonstrated by ELISA. ${ }^{* *} \mathrm{P} \leq 0.01$. (B) The amount of TGF- $\beta 1$ was demonstrated by ELISA using the supernatant of co-cultured tumor cells $\left(1.5 \times 10^{6}\right.$ cells/dish) and AEC IIs $\left(5 \times 10^{3}\right.$ cells/dish) (Super-CTA) by the Transwell assay. (H) The proliferative potential of MCF-7 cells inspected by MTT assay under 2D and 3D culture conditions with Super-TDA (50\%, 3D-Super) pretreated with monensin (3D-Mo-Super) or LY2109761 (3D-LY-Super) for 5 days DTC, dormant tumor cell; Super-TDA, supernatant of the transdifferentiation of AEC IIs to AEC Is; Super-CTA, supernatant of co-cultured tumor cells and AEC IIs by Transwell assay; AEC IIs, type II alveolar epithelial cells; AEC Is, type I alveolar epithelial cells.
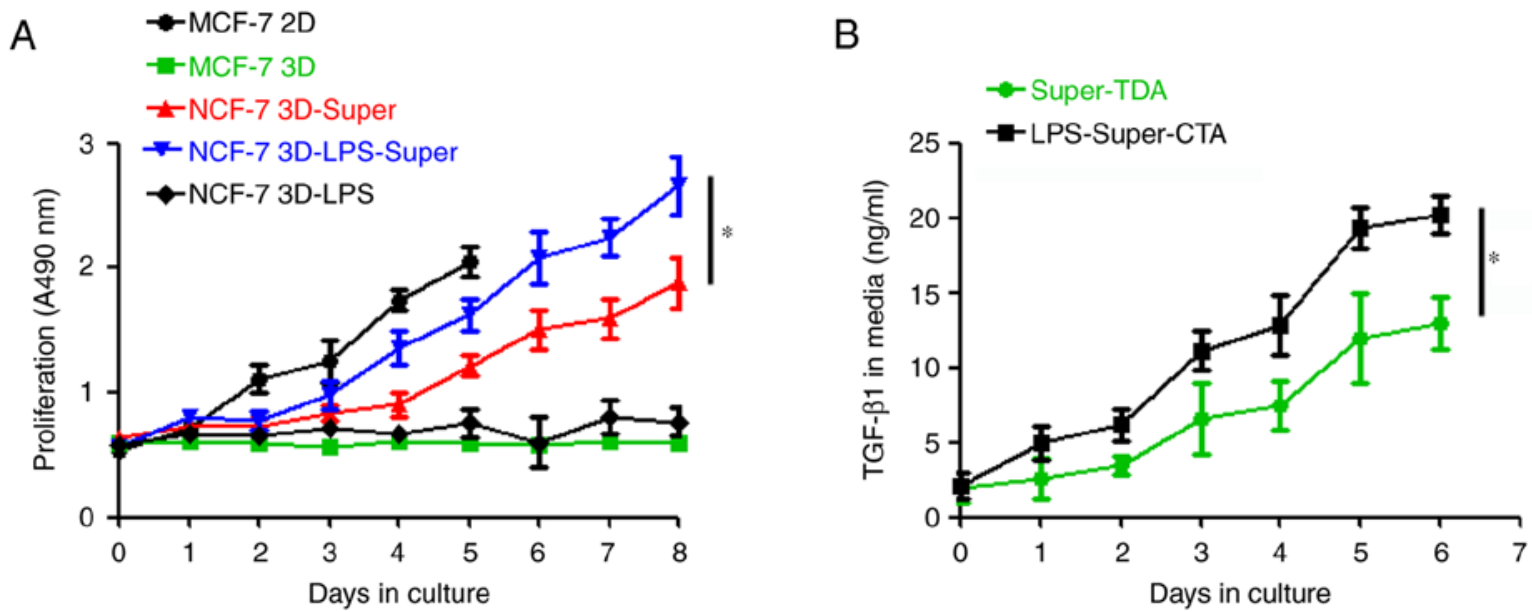

Figure 5. LPS promotes DTC reactivation by enhancing TGF- $\beta 1$ secretion in the Super-TDA. (A) Proliferative potential of MCF-7 cells tested by the MTT assay under 2D and 3D culture conditions. The 3D-established-DTCs of MCF-7 were stimulated with LPS alone (100 ng/ml) (MCF-7 3D-LPS), or Super-TDA, pretreated with LPS (MCF-7 3D-LPS-Super) or not pretreated (MCF-7 3D-Super). (B) Amount of TGF- $\beta 1$ in Super-TDA pretreated with LPS (LPS-Super-TDA) or not pretreated (Super-TDA) measured by ELISA. "P $\leq 0.05$. LPS lipopolysaccharide; DTC, dormant tumor cell; Super-TDA, supernatant of the transdifferentiation of AEC IIs to AEC Is; Super-CTA, supernatant of co-cultured tumor cells and AEC IIs by Transwell assay; AEC IIs, type II alveolar epithelial cells; AEC Is, type I alveolar epithelial cells.

of secreted TGF- $\beta 1$ increased markedly and more quickly when AEC IIs were co-cultured with tumor cells, suggesting that tumor cells enhanced the production and secretion of TGF- $\beta 1$ from AEC IIs during transdifferentiation (Fig. 4B). However, The secretion levels of other cell factors, such as TNF- $\alpha$, IL-1 $\beta 1$, IL-10, IL-6 and INF- $\gamma$, remained unchanged. $(\mathrm{P}>0.05)$ (Fig. 4B-G). Furthermore, the addition of the TGF- $\beta$ receptor kinase inhibitor LY2109761 or monensin inhibited the DTCs induced by Super-TDA (Fig. 4H), indicating that the autocrine effect of TGF- $\beta 1$ through transdifferentiation of AEC IIs is one of the major stimulators inducing DTC growth.
LPS stimulates the growth of DTCs by promoting the secretion of TGF- $\beta 1$ in the Super-TDA. TLR4 expressed on tumor cells has been found to contribute to tumor progression by promoting tumor cell proliferation, resistance to apoptosis and evasion from immune attack $(22,23)$. Therefore, we hypothesized that LPS may also participate in the activation of DTCs, and we used LPS to simulate inflammatory damage. We then incubated the DTCs with Super-TDA pretreated with or without LPS for several days, as shown in Fig. 5A. We found that although LPS could not induce DTC reproliferation directly, the reactivation and proliferation period of DTCs was obviously shortened to 2 days when the DTCs were co-cultured 

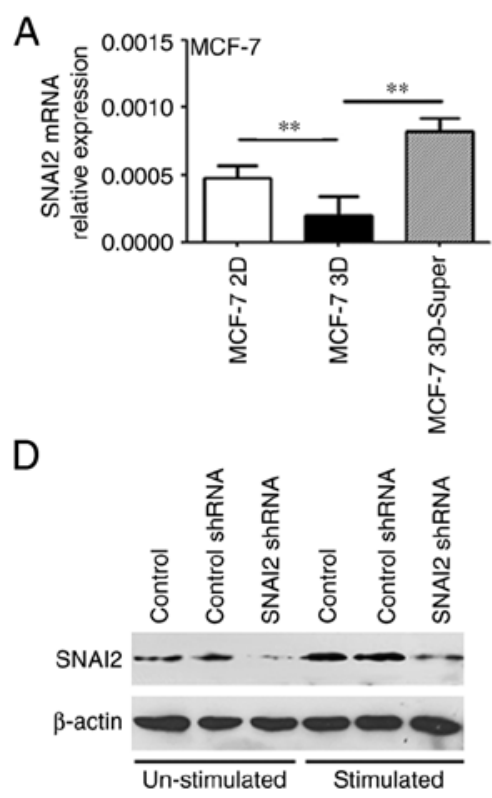

B

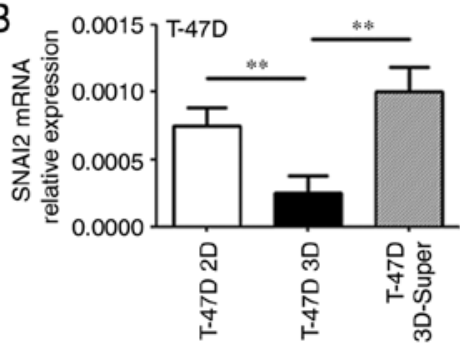

E

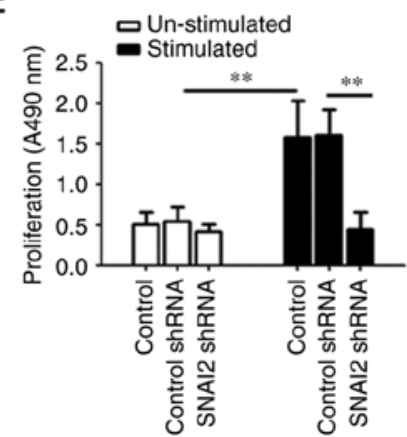

C

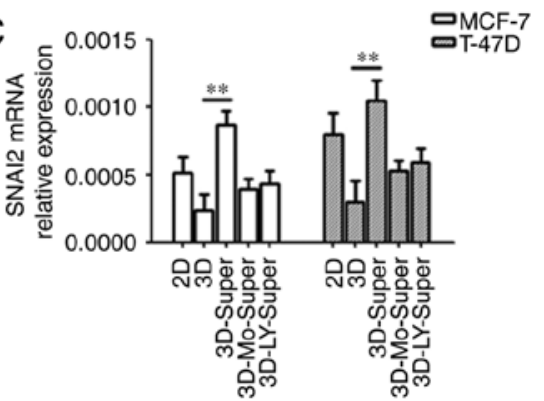

$\mathrm{F}$

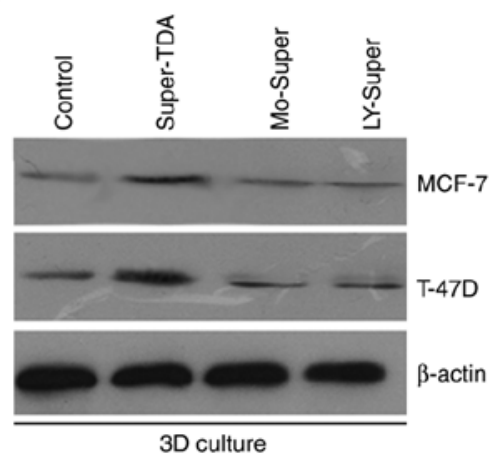

Figure 6. The transdifferentiation of AEC IIs promotes the upregulation of SNAI2 expression in reactivated-DTCs. The 3D-established-DTCs of MCF-7 and T-47D cells were cultured with Super-TDA (50\%, 3D-Super) pretreated with monensin (3D-Mo-Super) or LY2109761 (3D-LY-Super) for 5 days. SNAI2 expression was detected by (A-C) RT-qPCR and (F) western blotting in the indicated groups. ${ }^{*} \mathrm{P} \leq 0.05,{ }^{* *} \mathrm{P} \leq 0.01$. (D) Control MCF-7 cells and MCF-7 cells expressing control shRNA or SNAI2 shRNA were unstimulated (un-stimulated) or treated with Super-TDA (50\%, stimulated) for 5 days. (E) The proliferative potential of MCF-7 cells in a 3D culture system was detected via an MTT assay. AEC IIs, type II alveolar epithelial cells; AEC Is, type I alveolar epithelial cells; DTCs, dormant tumor cells; Super-TDA, supernatant of the transdifferentiation of AEC IIs to AEC Is.

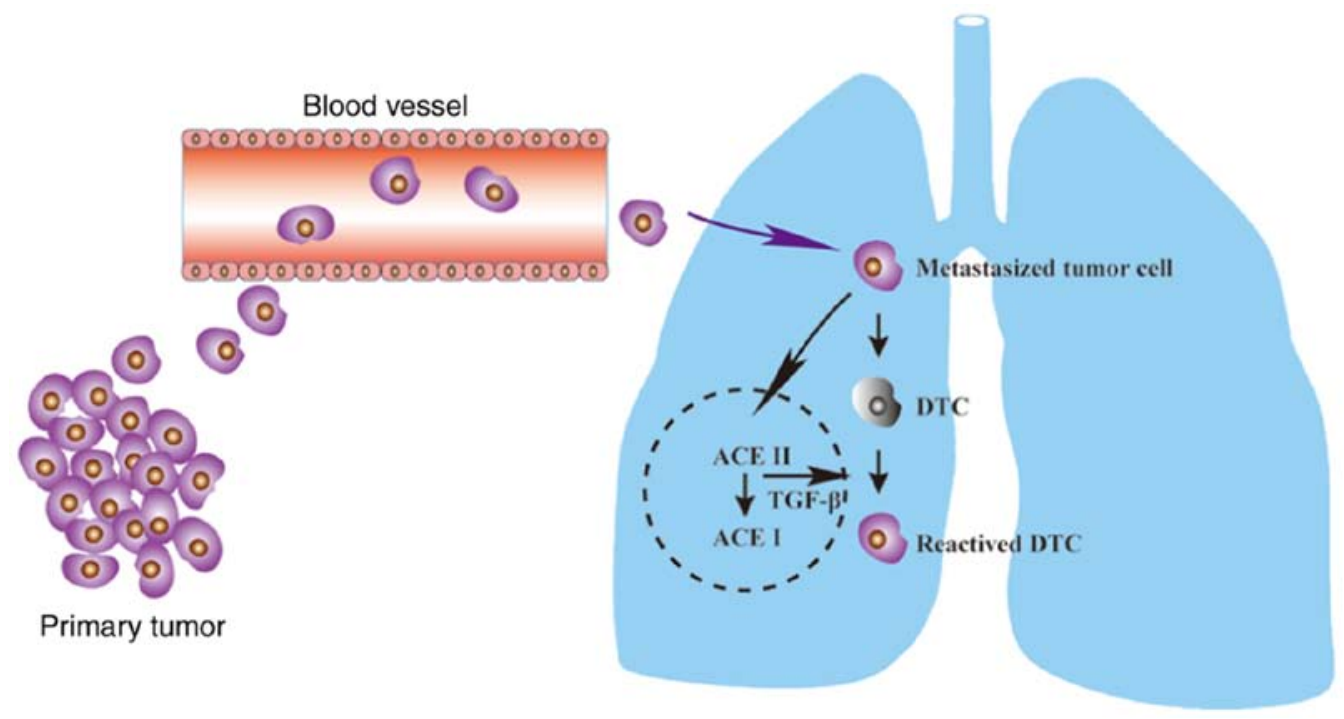

Figure 7. Association of AEC II transdifferentiation and the metastasis of tumor cells. Metastasized tumor cells may promote the transdifferentiation of AEC IIs into AEC Is, and AEC II transdifferentiation may in turn induce the metastasized DTCs switch to reactivated growth by enhancing TGF- $\beta 1$. AEC IIs, type II alveolar epithelial cells; AEC Is, type I alveolar epithelial cells; DTCs, dormant tumor cells.

with Super-TDA pretreated with LPS. Of note, the proliferative potential of the reactivated DTCs was obviously increased compared with that of the control groups, suggesting that LPS facilitates DTC growth. We also found that LPS promoted the release of TGF- $\beta 1$ from AEC IIs undergoing transdifferentiation (Fig. 5B), indicating that LPS may participate in the growth of DTCs by increasing the TGF- $\beta 1$ secretion from Super-TDA.
The transdifferentiation of AEC IIs promotes the upregulation of SNAI2 expression in the reactivated DTCs. SNAI2 serves an important role in the proliferation, metastasis and angiogenesis of tumors (24). SNAI2 enhances the kinase activity of cyclin D1/CDK4/CDK6, a central mediator in the transition from the $\mathrm{G} 1$ to the $\mathrm{S}$ phase, which further promotes the switch of tumor cell proliferation (25), indicating that SNAI2 may participate in the dormant-to-proliferating switch of DTCs. 
To confirm the role of SNAI2 in the dormant-to-proliferating switch of DTCs, we analyzed the dormant and reactivated tumor cells by qPCR and western blotting. As shown in Fig. 6A and B, Super-TDA promoted DTC upregulation of SNAI2 expression, and the upregulation of SNAI2 was inhibited by monensin or the TGF- $\beta$ receptor kinase inhibitor LY2109761 (Fig. 6C and F). We then used SNAI2 shRNA to inhibit the Super-TDA-induced increased expression of SNAI2 (Fig. 6D), which significantly hindered the re-proliferation of DTCs induced by Super-TDA (Fig. 6E), indicating that the upregulation of SNAI2 expression is required for the dormantto-proliferating switch of DTCs.

\section{Discussion}

Metastatic recurrence of carcinomas frequently occurs after a long latency period following removal of the primary tumor and administration of adjuvant therapy. Accumulating evidence suggests that tumor cells that have seeded to metastatic sites are resistant to conventional therapies, and remain dormant over long periods of time in target organs (26). A likely explanation of this phenomenon is that the presence of DTCs in secondary sites is maintained if the microenvironment is unfavorable for tumor cell proliferation $(3,4)$. However, it remains unclear whether tumor cells become dormant as a consequence of intrinsic defects, or in response to inhibitory signals that they encounter in foreign microenvironments.

Normal tissue homeostasis is maintained by signals from cell-cell communication, cell-cell adhesion and cell-matrix interactions, as well as more systemic mechanisms (e.g. hormones, cytokines) of growth and differentiation control (27). When the tumor microenvironment is disturbed, it may stimulate DTCs to resume active growth and progression; otherwise, they would remain dormant in the secondary target site $(3,28)$. The lung is one of the most common sites of metastasis (8). Our previous and other studies demonstrated that the metastatic tumor cells derived from non-invasive tumor cells exhibit lower expression of the proliferation marker Ki-67 (5). It is well-known that the new microenvironment may restrict the proliferation of disseminated tumor cells (38). Different from highly invasive tumor cells, the metastatic tumor cells derived from non-invasive tumor cells may be more sensitive to the restrictions of the new microenvironment.

In the present study, we demonstrated that tumor cells may promote the transdifferentiation of AEC IIs. Of note, the transdifferentiation of AEC IIs may in turn induce a DTC switch to reactivated growth by enhancing TGF- $\beta 1 /$ SNAI2 signaling (Fig. 7). Targeting this process may provide novel therapeutic strategies for inhibition of the dormant-to-proliferating metastatic cell switch.

AEC IIs have a multifunctional role in the lung, including secretory, synthetic and remodeling reservoirs for the lung epithelium to host defense (9). The progenitor function of AEC IIs is activated when the lung epithelium encounters a variety of conditions, including acute lung injury and inflammation, among others (10). AEC II proliferation and hyperplasia, followed by transdifferentiation, is a hallmark of alveolar epithelial injury, and defends the alveolus from injury (10). Apart from alveolar injury, the present study confirmed that the tumor cells also stimulated the transdifferentiation of
AEC IIs into AEC Is and TGF- $\beta 1$ secretion, suggesting that the metastasized tumor cells may participate in the induction of the transdifferentiation of AEC IIs to AEC Is in vivo, and AEC II transdifferentiation may be a common occurrence during lung metastasis.

In addition, AEC II transdifferentiation may in turn induce the DTC dormant-to-proliferating switch, which may explain why the lung is one of most common metastatic and recurrence sites (9); in addition, the metastasized tumor cells may also affect the microenvironment of the metastatic sites, which may be correlated with the dormant or proliferative behavior of tumor cells at the metastatic sites in vivo.

TGF- $\beta 1$, produced by AECs, is secreted during the transdifferentiation of AEC IIs to AEC Is, and the autocrine production of TGF- $\beta 1$ has been shown to promote the transdifferentiation of primary rat AEC IIs to AEC Is in vitro $(11,12)$. An autocrine loop of TGF- $\beta 1$ in mammary epithelium of pregnant mice has been shown to aid the differentiation process (29). The level of autocrine TGF- $\beta 1$ was found to be markedly increased when the AEC IIs were undergoing transdifferentiation phase, as shown by ours and other data $(13,29)$. Notably, the amount of TGF- $\beta 1$ during transdifferentiation of AEC IIs was 12-times higher than the basic secretion level in the present study, suggesting that the amount of TGF- $\beta 1$ secreted by AEC IIs during transdifferentiation may be sufficient to alter the tumor microenvironment and cause a variety of biological responses, including cell proliferation (14). In addition, both monensin and LY2109761 were able to block the dormant-toproliferating switch for DTCs induced by Super-TDA, further indicating that the TGF- $\beta 1$ from Super-TDA may be the main stimulator of DTC reactivation and proliferation in the lung.

The progenitor function of AEC IIs is activated when the lung epithelium encounters a variety of conditions, including acute lung injury and inflammation (10). TLR4 ligands (LPS) may be present in vivo due to surgery, cell damage, or the presence of bacteria in the tumor (30-32). TLR4 expressed on tumor cells has been found to contribute to tumor progression by promoting tumor cell proliferation, resistance to apoptosis and tumor evasion from immune surveillance $(22,23)$. Our results confirmed that LPS could not induce DTC reactivation directly, but rather by enhancing the secretion of TGF- $\beta 1$ from AEC IIs undergoing transdifferentiation, suggesting that inflammatory damage of the lung may participate in enhancing the reactivation process of DTCs. This may explain the fact that, when the lung epithelium encounters a variety of conditions, including acute lung injury, the progenitor function of AEC IIs is activated (10), promoting self-repair as well as DTC reactivation at the same time.

Although the expression of SNAI2 is very low in DTCs, the proliferative potential of DTCs was reactivated along with the increased expression of SNAI2 induced by Super-TDA. In addition, the upregulation of SNAI2 expression was inhibited by pretreatment with monensin and LY2109761 during the transdifferentiation of AEC IIs. The increased expression of SNAI2 is a major risk factors for the tumor recurrence and metastasis $(33,34)$. SNAI2 may enhance the kinase activity of cyclin D1/CDK4/CDK6, a central mediator in the transition from the $\mathrm{G} 1$ to the $\mathrm{S}$ phase, which further promotes the proliferation switch of tumor cells (25). Thus, SNAI2 may serve a pivotal role in the dormant-to-proliferating switch of DTCs. 
In summary, tumor cell metastasis to the lung may promote the transdifferentiation of AEC IIs to AEC Is in vitro. In addition, Super-TDA may in turn induce the reactivation of DTCs by enhancing TGF- $\beta 1 /$ SNAI 2 signaling. Recurrence and metastasis are responsible for the majority of cancer deaths. Given that the transdifferentiation of AEC IIs and the enhanced expression of TGF- $\beta 1 /$ SNAI2 are required for inducing the reactivation and proliferation of DTCs, targeting one of these stimuli or signaling pathways may be a viable approach to a comprehensive strategy for cancer treatment.

\section{Acknowledgements}

We would like to thank Professor Changyi Xiao, Dr Xiaokun Tu and Wei Wang for technical assistance in our experiments. We acknowledge research funding from the National Nature Science Foundation of China (grant nos. 81403163 and 81402404) and Yi Chang Scientific and Technological Bureau (grant nos. A14301-04 and A14301-10).

\section{Competing interests}

The authors declare that they have no competing interests.

\section{References}

1. Goss PE and Chambers AF: Does tumour dormancy offer a therapeutic target? Nat Rev Cancer 10: 871-877, 2010.

2. Páez D, Labonte MJ, Bohanes P, Zhang W, Benhanim L, Ning Y, Wakatsuki T, Loupakis F and Lenz HJ: Cancer dormancy: A model of early dissemination and late cancerrecurrence. Clin Cancer Res 18: 645-653, 2012.

3. Zappalà G, McDonald PG and Cole SW: Tumor dormancy and the neuroendocrine system: An undisclosed connection? Cancer Metastasis Rev 32: 189-200, 2013.

4. Alizadeh AM, Shiri S and Farsinejad S: Metastasis review: From bench to bedside. Tumour Biol 35: 8483-8523, 2014.

5. Zhou YH, Liao SJ, Li D, Luo J, Wei JJ, Yan B, Sun R, Shu Y, Wang Q, Zhang GM and Feng ZH: TLR4 ligand/ $\mathrm{H}_{2} \mathrm{O}_{2}$ enhances TGF- $\beta 1$ signaling to induce metastatic potential of non-invasive breast cancer cells by activating non-Smad pathways. PLoS One 8: e65906, 2013.

6. Barkan D, Kleinman H, Simmons JL, Asmussen H, Kamaraju AK, Hoenorhoff MJ, Liu ZY, Costes SV, Cho EH, Lockett S, et al: Inhibition of metastatic outgrowth from single dormant tumor cells by targeting the cytoskeleton. Cancer Res 68: 6241-6250, 2008.

7. Aguirre-Ghiso JA: Models, mechanisms and clinical evidence for cancer dormancy. Nat Rev Cancer 7: 834-846, 2007.

8. Minn AJ, Gupta GP, Siegel PM, Bos PD, Shu W, Giri DD, Viale A, Olshen AB, Gerald WL and Massagué J: Genes that mediate breast cancer metastasis to lung. Nature 436: 518-524, 2005.

9. Warburton D and Bellusci S: The molecular genetics of lung morphogenesis and injury repair. Paediatr Respir Rev 5 (Suppl A): S283-S287, 2004.

10. Fehrenbach H: Alveolar epithelial type II cell: Defender of the alveolus revisited. Respir Res 2: 33-46, 2001.

11. Xu W, Xu B, Zhao Y, Yang N, Liu C, Wen G and Zhang B: Wnt5a reverses the inhibitory effect of hyperoxia on transdifferentiation of alveolar epithelial type II cells to type I cells. J Physiol Biochem 71: 823-838, 2015.

12. Barkauskas CE, Cronce MJ, Rackley CR, Bowie EJ, Keene DR, Stripp BR, Randell SH, Noble PW and Hogan BL: Type 2 alveolar cells are stem cells in adult lung. J Clin Invest 123: 3025-3036, 2013.

13. Zhao L, Yee M and O'Reilly MA: Transdifferentiation of alveolar epithelial type II to type I cells is controlled by opposing TGF- $\beta$ and BMP signaling. Am J Physiol Lung Cell Mol Physiol 305: L409-L418, 2013.
14. Catalano V, Turdo A, Di Franco S, Dieli F, Todaro M and Stassi G: Tumor and its microenvironment: A synergistic interplay. Semin Cancer Biol 23: 522-532, 2013.

15. Liao SJ, Zhou YH, Yuan Y, Li D, Wu FH, Wang Q, Zhu JH, Yan B, Wei JJ, Zhang GM and Feng ZH: Triggering of Toll-like receptor 4 on metastatic breast cancer cells promotes $\alpha v \beta 3$-mediated adhesion and invasive migration. Breast Cancer Res Treat 133: 853-863, 2012.

16. Yan YL, Zhou YH and Ye H: An improved method for the isolation and primary culture of mouse AEC II. Hainan Med J 27: 3280-3282, 2016.

17. Zhang L, Zhao S, Yuan L, Wu H, Jiang H and Luo G: Hyperoxia-mediated LC3B activation contributes to the impaired transdifferentiation of type II alveolar epithelial cells (AECIIs) to type I cells (AECIs). Clin Exp Pharmacol Physiol 43: 834-843, 2016.

18. Isakson BE, Lubman RL, Seedorf GJ and Boitano S: Modulation of pulmonary alveolar type II cell phenotype and communication by extracellular matrix and KGF. Am J Physiol Cell Physiol 281: C1291-C1299, 2001

19. Almog N: Molecular mechanisms underlying tumor dormancy. Cancer Lett 294: 139-146, 2010.

20. Goss P, Allan AL, Rodenhiser DI, Foster PJ and Chambers AF: New clinical and experimental approaches for studying tumor dormancy: Does tumor dormancy offer a therapeutic target? APMIS 116: 552-568, 2008.

21. Weidenfeld K, Schif-Zuck S, Abu-Tayeh H, Kang K, Kessler O, Weissmann M, Neufeld G and Barkan D: Dormant tumor cells expressing LOXL2 acquire a stem-like phenotype mediating their transition to proliferative growth. Oncotarget 7: 71362-71377, 2016.

22. Huang B, Zhao J, Li H, He KL, Chen Y, Chen SH, Mayer L, Unkeless JC and Xiong H: Toll-like receptors on tumor cells facilitate evasion of immune surveillance. Cancer Res 65: 5009$5014,2005$.

23. Wang EL, Qian ZR, Nakasono M, Tanahashi T, Yoshimoto K, Bando Y, Kudo E, Shimada M and Sano T: High expression of Toll-like receptor $4 /$ myeloid differentiation factor 88 signals correlates with poor prognosis in colorectal cancer. Br J Cancer 102: 908-915, 2010.

24. Shih JY and Yang PC: The EMT regulator SNAI2 and lung carcinogenesis. Carcinogenesis 32: 1299-1304, 2011.

25. Casimiro MC, Velasco-Velázquez M, Aguirre-Alvarado C and Pestell RG: Overview of cyclins D1 function in cancer and the CDK inhibitor landscape: Past and present. Expert Opin Investig Drugs 23: 295-304, 2014.

26. Gao H, Chakraborty G, Lee-Lim AP, Mo Q, Decker M, Vonica A, Shen R, Brogi E, Brivanlou AH and Giancotti FG: The BMP inhibitor Coco reactivates breast cancer cells at lung metastatic sites. Cell 150: 764-779, 2012.

27. Glick AB and Yuspa SH: Tissue homeostasis and the control of the neoplastic phenotype in epithelial cancers. Semin Cancer Biol 15: 75-83, 2005.

28. Friberg S and Nyström A: Cancer metastases: Early dissemination and late recurrences. Cancer Growth Metastasis 8: 43-49, 2015.

29. Bhaskaran M, Kolliputi N, Wang Y, Gou D, Chintagari NR and Liu L: Trans-differentiation of alveolar epithelial type II cells to type I cells involves autocrine signaling by transforming growth factor beta 1 through the Smad pathway. J Biol Chem 282: 3968-3976, 2007.

30. Pidgeon GP, Harmey JH, Kay E, Da Costa M, Redmond HP and Bouchier-Hayes DJ: The role of endotoxin/lipopolysaccharide in surgically induced tumour growth in a murine model of metastatic disease. Br J Cancer 81: 1311-1317, 1999.

31. Tsan MF and Gao B: Endogenous ligands of Toll-like receptors. J Leukoc Biol 76: 514-519, 2004

32. Liu L, Yang M, Kang R, Wang Z, Zhao Y, Yu Y, Xie M, Yin X, Livesey KM, Loze MT, et al: DAMP-mediated autophagy contributes to drug resistance. Autophagy 7: 112-114, 2011.

33. Cheng WY, Kandel JJ, Yamashiro DJ, Canoll P and Anastassiou D: A multi-cancer mesenchymal transition gene expression signature is associated with prolonged time to recurrence in glioblastoma. PLoS One 7: e34705, 2012.

34. Hasan M, Sharma R and Saraya A: Slug is a predictor of poor prognosis in esophageal squamous cell carcinoma patients. PLoS One 8: e82846, 2013. 